\title{
Inferring and Removing a Spurious Response in the Optical Backscattering Signal from an Autonomous Profiling Float
}

\author{
BOŻENA WOJTASIEWICZ ${ }^{\mathrm{a}}$ \\ Indian Ocean Marine Research Centre, CSIRO Oceans and Atmosphere, Crawley, Western Australia, Australia, \\ and Institute of Oceanography, University of Gdańsk, Gdańsk, Poland \\ IAN D. WALSH \\ Sea-Bird Scientific, Philomath, Oregon \\ DAVID ANTOINE \\ Remote Sensing and Satellite Research Group, School of Earth and Planetary Sciences, Curtin University, Perth, \\ Western Australia, Australia, and Sorbonne Universités, UPMC Univ Paris 06, INSU-CNRS, Laboratoire d'Océanographie \\ de Villefranche, Villefranche-sur-mer, France
}

DiRK SLAWINSKI AND NiCK J. HARDMAN-MOUNTFORD

Indian Ocean Marine Research Centre, CSIRO Oceans and Atmosphere, Crawley, Western Australia, Australia

(Manuscript received 19 February 2018, in final form 19 July 2018)

\begin{abstract}
Development of autonomous profiling floats, allowing for long-term continuous measurement of biooptical variables, promises to significantly increase our knowledge of the variability of the particulate optical backscattering coefficient $b_{\mathrm{bp}}$ in marine environments. However, because autonomous floats are designed for unattended data collection and only rarely are recovered for analysis of the condition of the sensors in situ, the quality-control analysis of float data streams is of paramount importance in both a particular dataset and the larger understanding of the response of sensors over the lifetime of the floats. Anomalous data from a backscattering sensor (MCOMS) mounted on autonomous profiling floats are reported here. The observed sensor behavior, which presents itself as significant differences in the values observed at the parking depth between profiles, caused by a steady increase in the signal during the profiling time, is neither common to all sensors nor can it be a function of changes in the particle population. A simple quality-control procedure that is able to detect this spurious sensor response is proposed. Further characterization of this effect will require laboratory experimentation under controlled conditions of temperature and pressure.
\end{abstract}

\section{Introduction}

Light transmittance in the ocean is modulated by the presence of dissolved materials and particles, which include biological, mineral, and detrital materials, as well as air bubbles from breaking waves and gas bubbles from seeps (e.g., Stramski et al. 2004; Antoine et al. 2011).

\footnotetext{
${ }^{\text {a }}$ Current affiliation: CSIRO Oceans and Atmosphere, Hobart, Tasmania, Australia.

Corresponding author: Bożena Wojtasiewicz, bozena.wojtasiewicz@ csiro.au
}

In most of the open ocean, biological processes dominate the particle field and light transmittance (as the beam attenuation coefficient) is linearly correlated with the particle mass concentration (Gardner et al. 1993; Walsh et al. 1995; Cetinić et al. 2012). The beam attenuation coefficient from single-wavelength transmissometers has been used to study particle dynamics in the ocean, primarily from shipboard profiling systems (e.g., Bricaud et al. 1995). Higher uncertainty occurs when deriving particle mass concentration from instruments measuring optical backscattering, which will be referred to as backscattering for brevity, because the position of a given particle relative to the 
optical field, as well as the particles' composition, are significant to the measurement and degrade the relationship between particle mass concentration and the optical signal (Cetinić et al. 2012). The backscattering measurement has, however, an advantage for long-term monitoring on autonomous vehicles over the beam attenuation measurement, because backscattering sensors are smaller and require less power compared to transmissometers. The backscattering coefficient measured in situ can also be directly compared to its value derivable from satellite ocean color observations, which cannot be done for the beam attenuation coefficient. While the backscattering measurement is a function of the composition of the particle field and the particle size distribution, it differs from the beam attenuation measurement in that the uniformity of the particle field and the position of an individual particle relative to the sensor is reflected in the data. This difference results in a less robust correlation between particle mass concentration and particulate backscattering $b_{\mathrm{bp}}$, but it also yields information on the particle size distribution, especially of the larger particles (Briggs et al. 2011). This means that backscattering sensors have the potential to deliver a key component to models of the vertical particle flux, which is itself critical to understanding carbon sequestration in the ocean.

Considering the advantages of the autonomous float data, one needs to understand that autonomous measurements that are taken without operator control pose new challenges in terms of data quality control and management. Sensors' characterizations are provided by manufacturers and can be complemented by CTD and bio-optical casts performed at the time of deployment. This results in a sparse set of control data relative to the total data stream. It is therefore necessary to assess the measurement uncertainty and to characterize all possible sources of errors, especially for measurements performed in open-ocean oligotrophic waters, where the particle mass concentration is low, resulting in measurements close to the resolution of most backscattering instruments. Uncertainty in $b_{\mathrm{bp}}$ measurements in oligotrophic waters may be as high as $40 \%$ (Dall'Olmo et al. 2012) near the limit of detection.

For oligotrophic environments, effective measurements of backscattering will have to account for small variances caused by the sensors, which may otherwise be overlooked in more particle-rich environments. The aim of this paper is to present and characterize an anomaly in data from the MCOMS backscattering sensor mounted on some types of biogeochemical (BGC) Argo floats (Navis, Sea-Bird). The anomaly can substantially affect the $b_{\mathrm{bp}}$ values; thus, it needs to be accounted for during the quality-control process for BGC-Argo backscattering profiles and uncertainty estimations. Our results suggest a technical basis for the anomaly, and we propose a simple detection method. Such a sensor behavior has not been previously reported in studies using different types of backscattering sensors (e.g., Boss et al. 2008; Green et al. 2014; Dall'Olmo and Mork 2014; Mignot et al. 2014), so it is unclear whether the anomaly applies only to one model of sensor or has become noticeable only from comparative analysis of deep and shallow backscattering profiles enabled by the flexible mission programming of BGC-Argo floats.

\section{Instruments and data}

Four autonomous profiling floats (Navis BGCi, SeaBird Scientific, Inc., Bellevue, Washington) were deployed in July 2015 in the Indian Ocean subtropical gyre. Another two floats were deployed in January 2016 in the southern Indian Ocean (Fig. 1). In addition to standard Argo CTD sensors (SBE41-CP, Sea-Bird), all floats were equipped with an SBE63 oxygen optode (SeaBird), a three-channel MCOMS (WET Laboratories, Inc., Philomath, Oregon) sensor measuring chlorophyll $a$ fluorescence and the volume scattering function $\beta$ at $149^{\circ}$ and at two wavelengths (532 and $700 \mathrm{~nm}$ ) (floats $387,388,390,391,528)$, or chlorophyll $a$ and colored dissolved organic matter (CDOM) fluorescence and $\beta$ at $149^{\circ}$ and at one wavelength $(700 \mathrm{~nm})$ (float 392). The volume scattering function measured by both types of sensors is a sum of the input from backscattering by particles and seawater; $\beta=\beta_{p}+\beta_{w}$. Calibration of all sensors was performed by the manufacturer before mounting them on the float. In this paper we will look only at the MCOMS optical backscattering data.

The MCOMS optical instrument is designed for deployment on long-lasting autonomous profiling floats. The housing is titanium with a $2000-\mathrm{m}$-depth rating. The optical windows are quartz and are bonded directly to the titanium faceplate of the instrument. There is an open volume between the windows and the rest of the optical assemblage in each bore hole, which means there are internal optical surfaces that will have some aging characteristics. No biofouling prevention methods are taken with the MCOMS apart from parking below the photic zone in relatively cold waters, which has been proven to be an effective method for preventing biofouling during prolonged deployments (e.g., Riser and Johnson 2008; Boss et al. 2008). The instrument is sealed under vacuum to minimize initial humidity and to demonstrate seal integrity. Connectivity to the float is through a custom-designed connector. Electronics are isolated from pressure and flexing of the housing to minimize stress on the components. Instruments are 


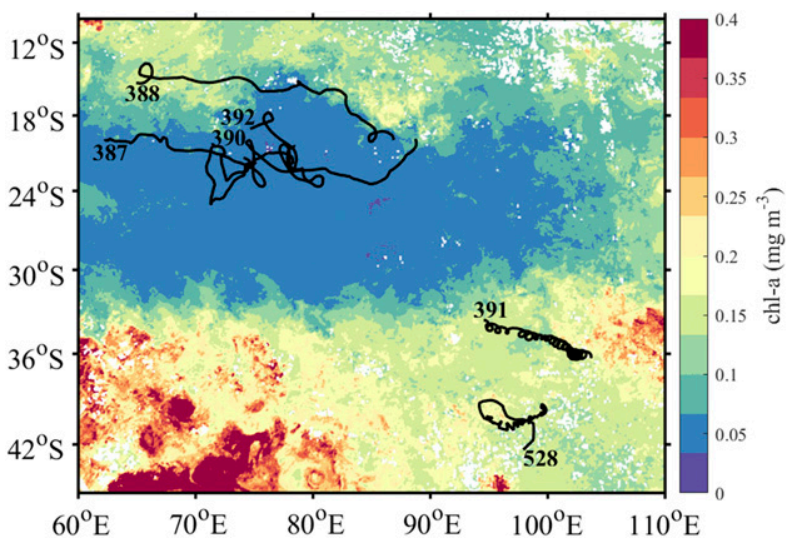

FIG. 1. Trajectories of floats deployed in the subtropical gyre $(387,388,390,392)$ and southern Indian Ocean $(391,528)$. The color shading corresponds to the satellite (MODIS) chlorophyll concentration $\left(\mathrm{mg} \mathrm{m}^{-3}\right)$ in October 2016.

stress tested (e.g., vibration, temperature, pressure) at the factory to maximize the likelihood of failure within the factory and to minimize failure in the field over the expected life of the instrument.

After each profile, the raw electronic counts from MCOMS were transmitted to land through the iridium antennas and were then converted into $\beta$ at the scattering angle $\theta_{c}$, which is $149^{\circ}$ for this sensor, and selected wavelength $\lambda$ using the factory-provided scale factor (SF) and dark count (DC) values. The dark counts provided by the manufacturer were used in all cases because the DC was measured only immediately prior to float deployment with black electrical tape covering the sensor for the southern Indian Ocean deployments, and the difference between the measured and factory DC was fewer than two counts, which corresponds to a backscattering coefficient between 1.0 and $1.4 \times$ $10^{-5} \mathrm{~m}^{-1}$ depending on the float because of differences in the magnitude of the scale factor.

As mentioned above, the volume scattering function measured by the MCOMS sensor represents the sum contribution from the particles and the seawater. The volume scattering function of seawater itself at the chosen scattering angle and wavelength $\beta_{w}\left(\lambda, \theta_{c}\right)$ is calculated using the float temperature and salinity data according to the method described by Zhang et al. (2009) with a depolarization ratio of 0.039 . Then the particulate backscattering coefficient is calculated as

$$
b_{\mathrm{bp}}(\lambda)=2 \pi \chi\left[\beta\left(\lambda, \theta_{c}\right)-\beta_{w}\left(\lambda, \theta_{c}\right)\right]=2 \pi \chi\left[\beta_{p}\left(\lambda, \theta_{c}\right)\right]
$$

with $\chi=1.14$ when $\theta_{c}=149^{\circ}$ in the case of MCOMS sensor according to Sullivan et al. (2013).
Data were collected with a 2-m vertical resolution during the upward profile. The profile frequency and the parking depth varied among the floats and throughout the floats' life, depending on the programmed mission configurations. For example, float 387 was parked at $500 \mathrm{~m}$ at the beginning and profiled every $6 \mathrm{~h}$. After about 2 months of operation, the profiling frequency was changed to once a day and the parking depth was changed to $300 \mathrm{~m}$. Some of the profiles were performed from the parking depth to the surface and in some cases the float descended to 1000 or $2000 \mathrm{~m}$ and then profiled to the surface (Table 1).

\section{Results and discussion}

Because autonomous floats generate substantial data streams, multiple-dimension visualization plots (Fig. 2) and discernment of anomalies with the human eye are critical components of the quality-control (QC) process. Plots of raw counts from MCOMS backscattering sensors revealed a blocky feature associated with the time period when the floats profiled from 1000 or $2000 \mathrm{~m}$ relative to shallower profiles (from 300 or $500 \mathrm{~m}$ ). This feature represents itself as light- and dark-colored stripes associated with the depth at which profiling started. It can be clearly seen during the period when the float was profiling to alternating starting depths of 300 or $2000 \mathrm{~m}$ (since November 2015). All the shallow profiles can be seen as brighter stripes, indicating lower values measured by the sensor at the same depths compared to the deep profiles. This anomaly was pronounced only in the 532-nm backscattering data (Fig. 2a) and not in the 700-nm data (Fig. 2b). When looking at individual profiles, the offset between deep and shallow profiles was even more evident (Fig. 3). The average initial profile value, calculated as the median of the five deepest measurements for each profile, measured by float 387 was consistent across the entire time series. For example, during the post-November 2015 period with alternating shallow $(\sim 300 \mathrm{~m})$ and deep $(\sim 2000 \mathrm{~m})$ profiles, the average plus and minus one standard deviation (SD) raw count value for the 532-nm channel on float 387 (resolution of 1.6 counts according to the calibration sheet) was $142.5 \pm 2.6$ and $143.5 \pm 1.2$ for shallow and deep profiles, respectively, and were not statistically different (Kruskal-Wallis test, $p=0.43$ ). Thus, this demonstrates that regardless of the starting depth (either 300 or $2000 \mathrm{~m}$ ), the sensor was always recording a similar initial profile value.

The digital counts returned by the MCOMS sensor consist of the background voltage, called the dark count, and the backscattering signal from the illuminated volume of seawater and particles. Particles are scarce in oligotrophic environments; therefore, the contribution 
TABLE 1. General information on the floats deployed in 2015-16 in the Indian Ocean. ID = identification.

\begin{tabular}{cccccc}
\hline \hline Float ID & WMO ID & Deployed & No. of profiles $^{\text {a }}$ & Max depth (m) $^{\text {Parking depth (m) }}$ \\
\hline 387 & 5904923 & 5 Jul 2015 & 295 & 2000 & 300 (initially 500) \\
388 & 5904924 & 6 Jul 2015 & 293 & 2000 & (initially 500) \\
390 & 1901347 & 8 Jul 2015 & 282 & 1000 & 300 (initially 500) \\
392 & 1901348 & 8 Jul 2015 & 228 & 1000 & 300 (initially 500) \\
391 & 5905022 & 11 Jan 2016 & 273 & 2000 & 400 \\
528 & 5905023 & 12 Jan 2016 & 248 & 400 \\
\hline
\end{tabular}

${ }^{\text {a }}$ By 21 Nov 2016.

of particles to the total output is low. In our data, at depths below the seasonal thermocline, the particle signal at $532 \mathrm{~nm}$ is only $\sim 10 \%$ of the output and even at the deep chlorophyll maximum (DCM) the particles contribute only $\sim 50 \%$ of the total output (Fig. 5). For example, in the case of float 387 , the factory dark count was 20 counts and the seawater molecular scattering was responsible for 105-112 counts, whereas particles were responsible for only about 40 counts in the mesopelagic zone and up to 126 counts in the surface layer (Fig. 5). The seawater scattering is calculated using temperature and salinity profiles (Zhang et al. 2009), and all the variations in the sensor behavior are contained in the magnitude of the particulate backscattering coefficient itself. Therefore, it is worth analyzing this problem, not only by looking at raw counts for the total backscattering function, but also using the derived magnitude of the particulate backscattering coefficient. Considering the post-November 2015 period of profiles (i.e., starting at the time indicated by the dashed line in Fig. 4) that were initiated from either $300 \mathrm{~m}$ (close to the park depth) or $2000 \mathrm{~m}$ (deep profile depth), the average $\pm 1 \mathrm{SD} b_{\text {bp }}(532)$ at $250-\mathrm{m}$ depth was $2.41 \times 10^{-4} \pm$ $0.45 \times 10^{-4}$ and $3.33 \times 10^{-4} \pm 0.36 \times 10^{-4} \mathrm{~m}^{-1}$, respectively. Such a difference was not observed for the other $(700 \mathrm{~nm})$ backscattering channel in this sensor, for which the average $b_{\mathrm{bp}}(700)$ for shallow and deep profiles were $2.37 \times 10^{-4} \pm 0.24 \times 10^{-4}$ and $2.53 \times 10^{-4} \pm 0.27 \times$ $10^{-4} \mathrm{~m}^{-1}$, respectively.

In this case, each time the float performs a profile, the measurements at parking depth are recorded at the beginning of the cycle. The float then descends to the programmed profile start depth and starts to record the measurements with preset frequency during its ascent (every $2 \mathrm{~m}$ in this case). We compared the parking-depth values recorded before the profile started $b_{\mathrm{bp} \_ \text {parking }}$ with the values at the same depth measured during the profile $b_{\text {bp_profile }}$ (Fig. 6). The measurements are only a few hours apart and were performed at about $300 \mathrm{~m}$, where the variability in the magnitude of the backscattering coefficient is rather low (with the exception of periods with high productivity in the surface layer-for example, December-February in the case of float 387, when a relatively high surface backscattering signal was still observed at 300-m depth), so we can assume that the values should be very similar. Additionally, we did not see any statistically significant differences (Wilcoxon signed-rank test) between temperature $(p<0.05)$ or
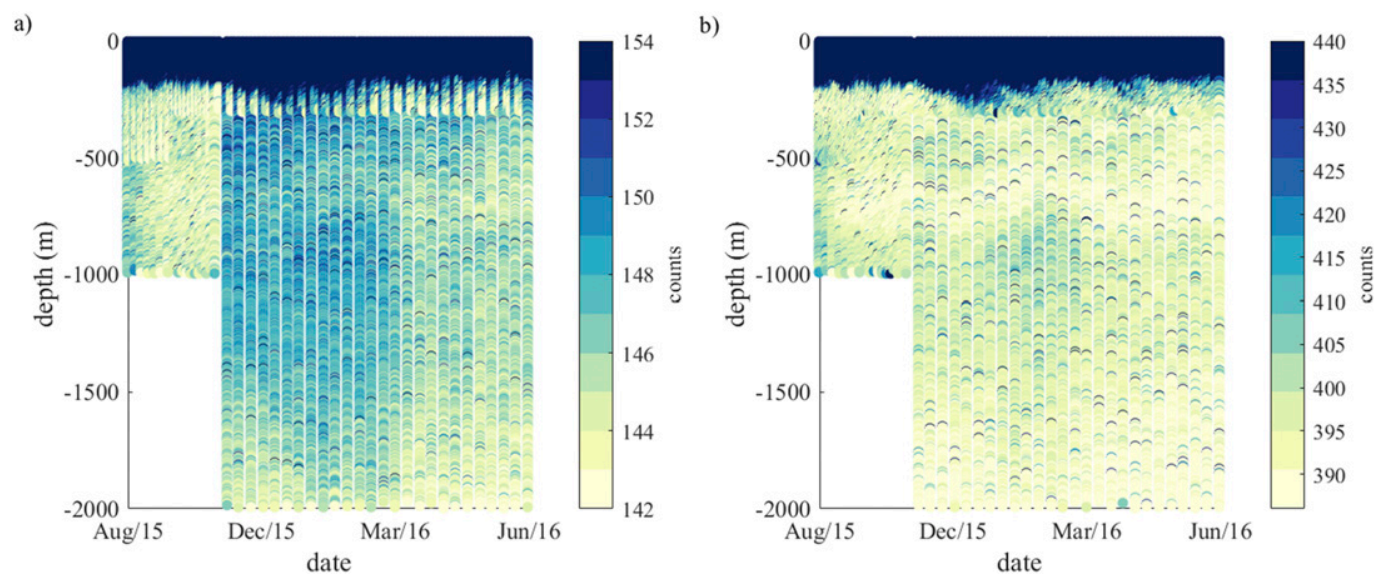

FIG. 2. Raw counts for total backscattering function $\beta$ at $149^{\circ}$ at (a) 532 and (b) $700 \mathrm{~nm}$ measured by the MCOMS sensor placed on float 387 . No interpolation was used to create these figures. 

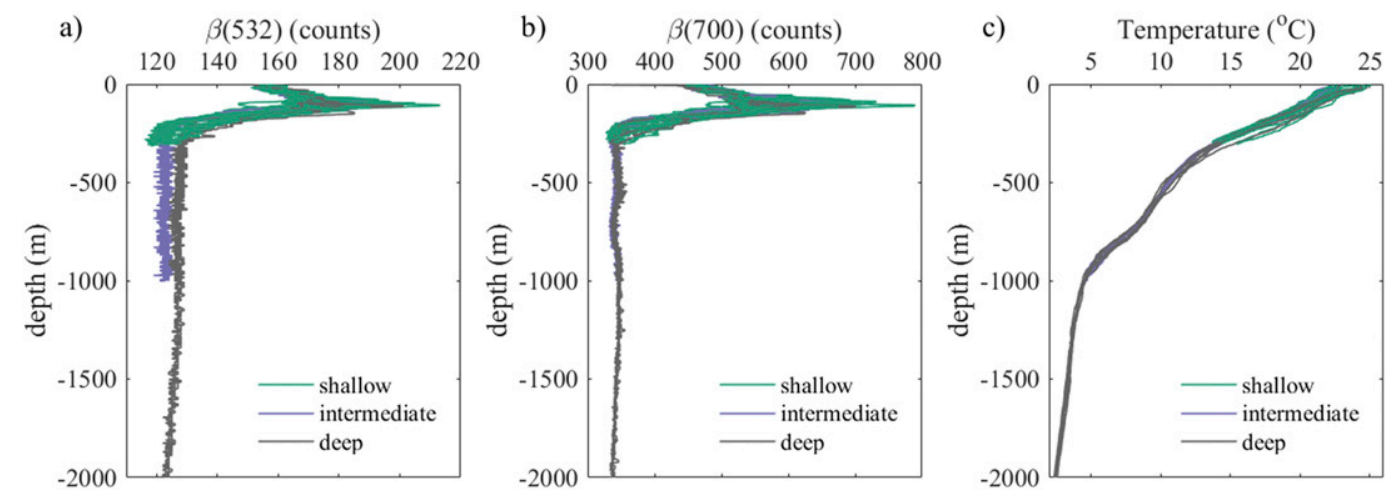

FIG. 3. Profiles of raw counts for total backscattering function at $149^{\circ}$ at (a) 532 and (b) $700 \mathrm{~nm}$, and (c) temperature measured by float 387 between 12 Oct and 14 Dec 2015. Different colors indicate profiles initiated at about 2000 (deep), 1000 (intermediate), and $300 \mathrm{~m}$ (shallow).

oxygen concentration $(p<0.01)$ recorded at the parking depth and during the profile. For all analyzed floats, apart from float 387 , the difference between $b_{\text {bp_parking }}$ and $b_{\text {bp_profile }}$ was not statistically different from $0(p<$ 0.05 ) (Fig. 7) and did not depend on the difference between the profile starting depth and the parking depth (Fig. 6a). Only for float 387 were the differences at the parking depth correlated with the distance traveled by the float before reaching the parking depth during the profile (Fig. 6a). These differences were also statistically different among shallow, intermediate, and deep profiles (Kruskal-Wallis test, $p<0.001$; Fig. 6b). For all shallow profiles (performed in the upper $600 \mathrm{~m}$ with a parking depth at either $\sim 500$ or $\sim 300 \mathrm{~m}$ ), the median difference between $b_{\text {bp_parking }}$ and $b_{\text {bp_profile }}$

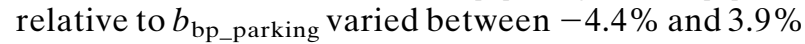
(Fig. 6). Apart from float 387 for which the median difference for deep profiles was $-63.7 \%$ (Fig. 6b), the median difference at the parking depth did not exceed $\pm 4.5 \%$ (Figs. $6 \mathrm{c}-\mathrm{f}$ ).

It is very hard to explain this behavior of the MCOMS sensor, as it was clearly detectable in only one out of five sensors we looked at (Fig. 6) and only in the 532-nm channel. The most probable cause is likely to be self-heating creating the steady increase of the recorded value, or a long acclimation of the sensor to the surrounding temperature, resulting in the initial values to be lower than the real ones, as the increase in $b_{\mathrm{bp}}$ correlates with time (hence voltage draw) from the profile start. Another possible reason for the different response of the sensor at various depths could be the change in the intensity of the light-emitting diode (LED) with changing water temperature, which changes with depth [see Fig. 6.7 in Sullivan et al. (2013)] with the response being different for the 532- and 700-nm LED. If the intensity of the LED at depths where water temperature is the lowest was lower than expected, then the signal detected by the receiver would be decreased compared to the true value. However, the correlation between the initial $b_{\mathrm{bp}}(532)$ value recorded by the sensor and the water temperature was very weak $(r=0.13 ; p=$ $0.034)$. In all these cases the spurious response of the sensor would be observed as the increase in the signal with decreasing depth (Fig. 6a). In the first case, the true value would be recorded at the beginning of

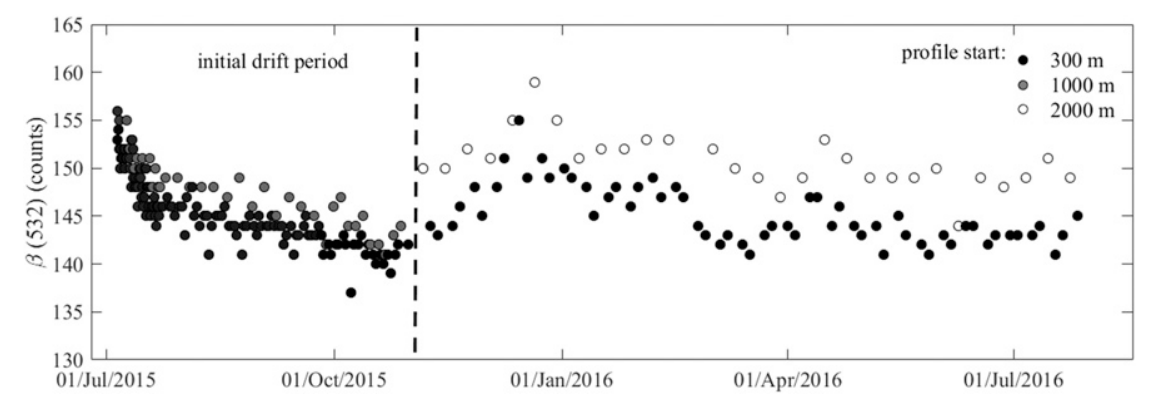

FIG. 4. Contribution of seawater and particulate backscattering to the signal measured by the MCOMS sensor. All presented values are raw counts. 


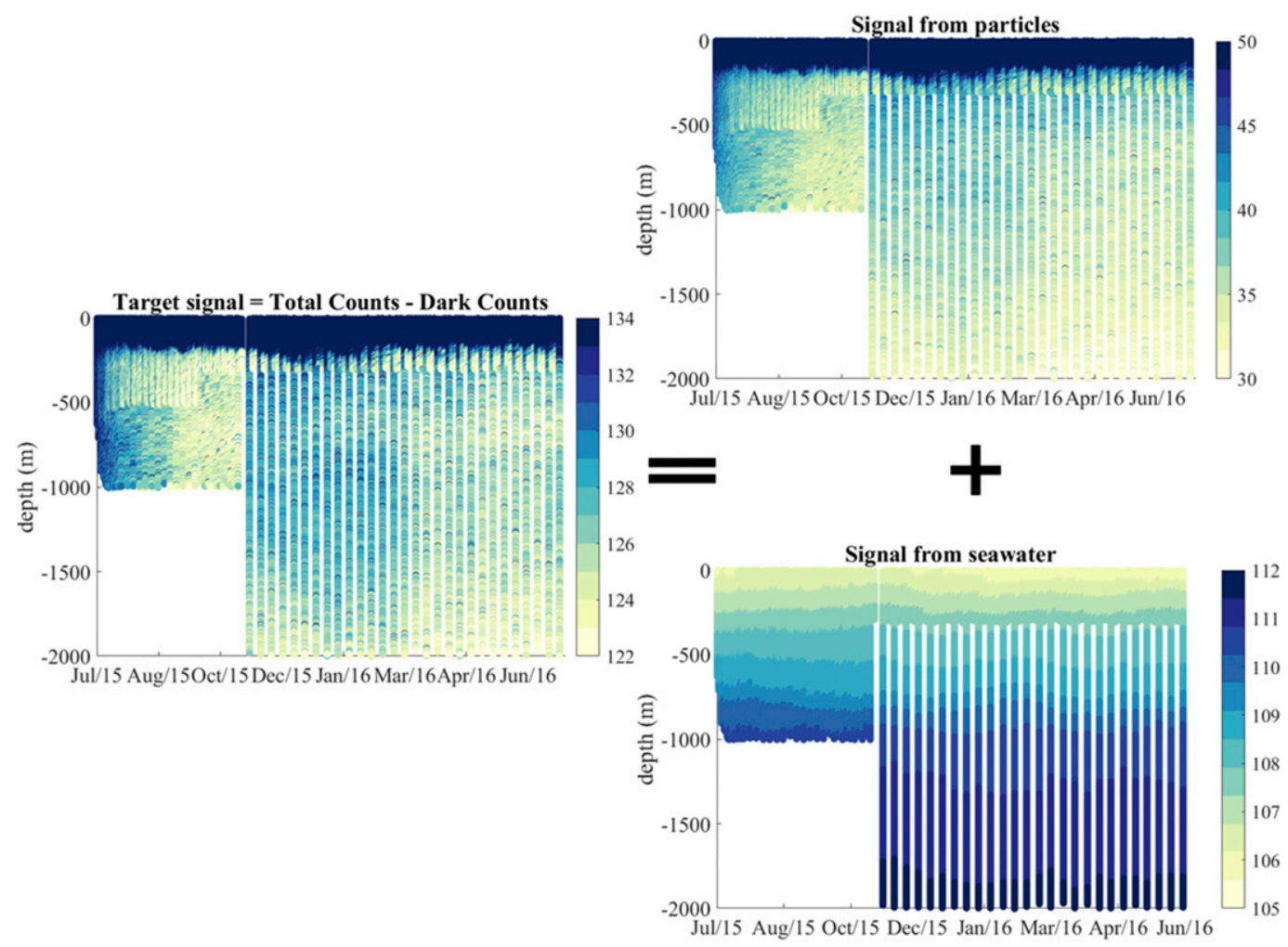

FIG. 5. Values of the particulate backscattering coefficient at $532 \mathrm{~nm}, b_{\mathrm{bp}}(532)$, at $250 \mathrm{dbar}$. Different colors indicate profiles initiated at about 2000 (deep), 1000 (intermediate), and $300 \mathrm{~m}$ (shallow).The dashed vertical line separates the part of the time series when we observed the initial drift, and the float was programmed to profile only down to $1000 \mathrm{~m}$ from the part when no drift in profile minimum value was observed and all profiles were either shallow ( $\sim 300$ dbar) or deep ( -2000 dbar).

the profile with the surface values mostly affected, whereas in the latter ones, the values recorded closer to the surface would be closer to the true value, which mostly would affect studies focusing on the mesopelagic carbon content and carbon export. However, the determination of the causative factor is impossible without conducting detailed laboratory tests. Regardless in all described cases, the failure in the performance of the sensor could be detected by a comparison of the parking depth values.

As mentioned before, only one of the backscattering sensors on the floats that we deployed during this project exhibited offsets between the deep and shallow profiles (Fig. 6). All other floats and sensor packages were coherent within the observed and expected ranges; that is, we did not observe any significant differences between deep and shallow profiles, and the median of the difference between the counts recorded at the parking depth before the commencement and during the profile did not exceed two counts, which is very close to the sensitivity limit of backscattering meters (Johnson et al. 2017). While it is tempting to simply ignore the data from the anomalous sensor, we considered that given the expense and uniqueness of the dataset, as well as the likelihood that individual sensor anomalies will occur again, it is at the least an instructive exercise to build a method to reduce the anomaly. To perform any kind of correction, the assumption on which of the measured values can be assumed as "true" needs to be undertaken. It has been previously observed that the particulate backscattering at $700 \mathrm{~nm}$ within the mesopelagic $(900-950 \mathrm{~m})$ is relatively constant (Poteau et al. 2017). We found relatively large differences among the values recorded within the mesopelagic by individual floats considered in this study at both 532 and $700 \mathrm{~nm}$. Before the deployment of each of these floats, an independent cast with the multispectral backscattering meter, Hydroscat-6 (Maffione and Dana 1997), was performed. This gave us an opportunity to calibrate all the MCOMS sensors to the Hydroscat-6 results [for more details see Wojtasiewicz et al. (2016)], which enabled us to compare the $b_{\mathrm{bp}}$ values recorded by each floats. We found that $b_{\mathrm{bp}}(700)$ 
a)

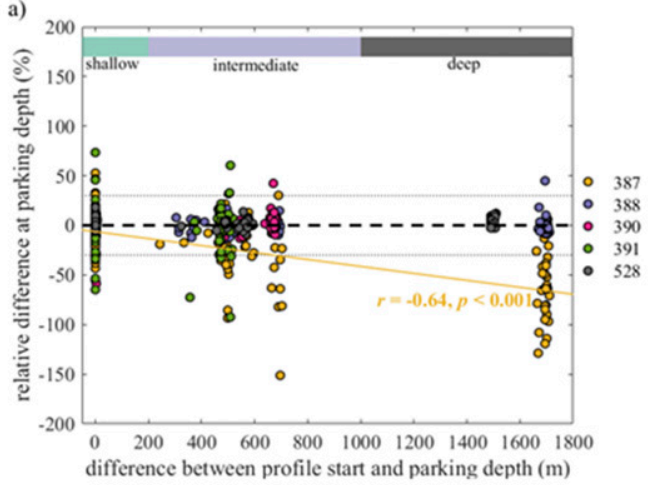

c)
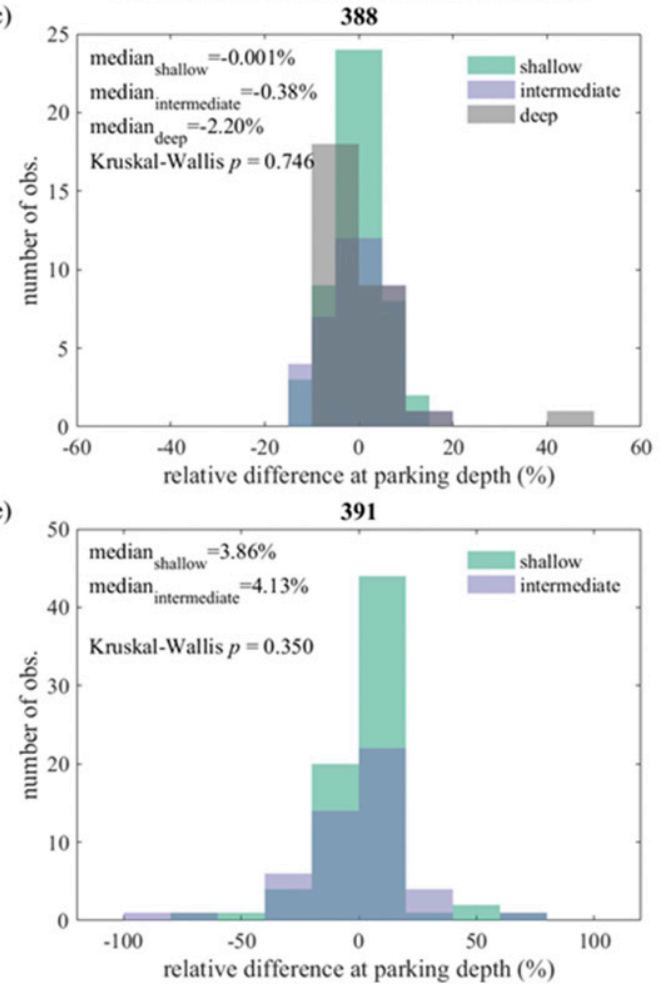

b)

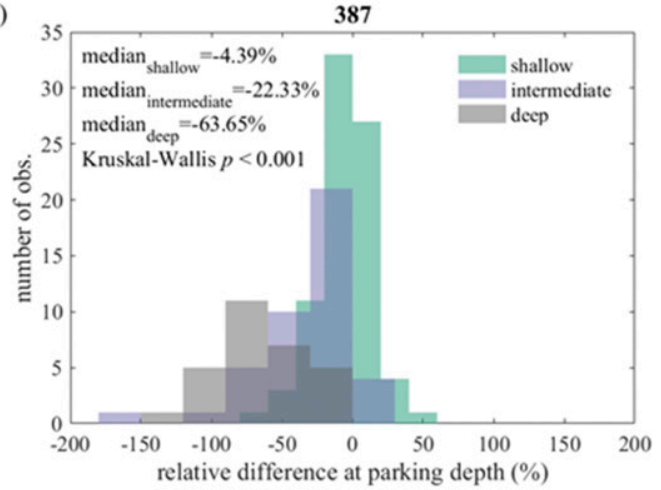

d)

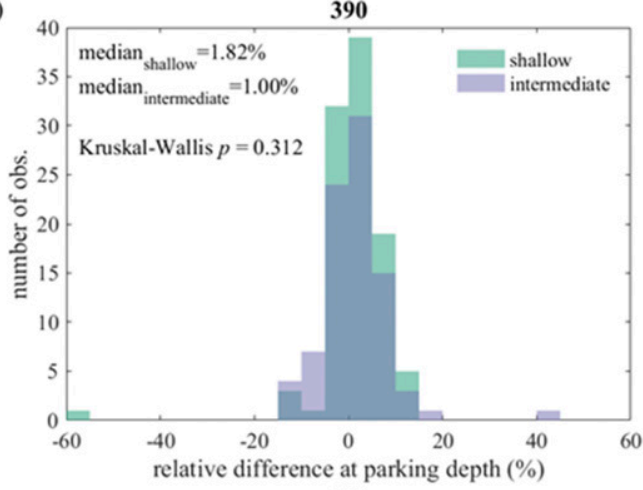

f)

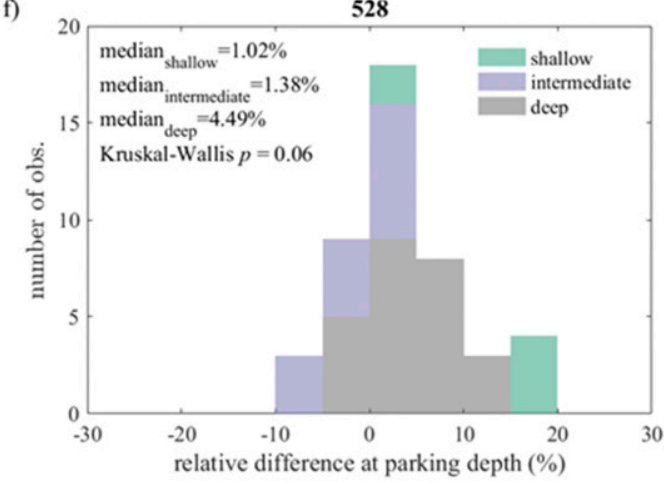

FIG. 6. Relative difference between the backscattering coefficient at $532 \mathrm{~nm}$ measured at the parking depth before $b_{\text {bp_parking }}$ and during the profile $b_{\text {bp_profile. }}$ (a) Dependence between the relative difference at the parking depth and the distance traveled by the float before reaching the parking depth. Black dashed and dotted lines indicate $0 \%$ and $\pm 30 \%$, respectively. Yellow dashed line indicates a statistically significant relationship ( $r=-0.64, p<0.001$ ) observed for float 387. (b)-(f) Histograms of relative differences at the parking depth for shallow $(<500 \mathrm{~m})$, intermediate $(-1000 \mathrm{~m})$, and deep profiles $(-2000 \mathrm{~m})$ for floats $387,388,390,391$, and 528 , respectively. Kruskal-Wallis $p$ values lower than 0.05 indicate statistically significant differences between shallow, intermediate, and deep profiles. For clarity, the $x$ axis ranges differ among the histograms.

measured between 900 and $950 \mathrm{~m}$ was relatively constant, whereas large variability in the $b_{\mathrm{bp}}(532)$ channel was noted. More similar results were seen at the 1950 2000-m-depth interval, but still the values recorded by individual floats were significantly different $(p<0.01)$ among each other. This inconsistency in the obtained results and the limited number of floats carrying the MCOMS sensor in this region do not allow confirmation of which hypothesis is correct.
The correction we applied was primarily designed to produce an internally consistent dataset by removing the spurious vertical trend difference between shallow and deep profiles. As such, this correction should allow for analysis of the temporal and vertical structure of changes in the $b_{\mathrm{bp}}$ record. The assumptions made in the processing are pragmatic and reasonable, although they may require further refinement as new observations improve our understanding of either a deep baseline 

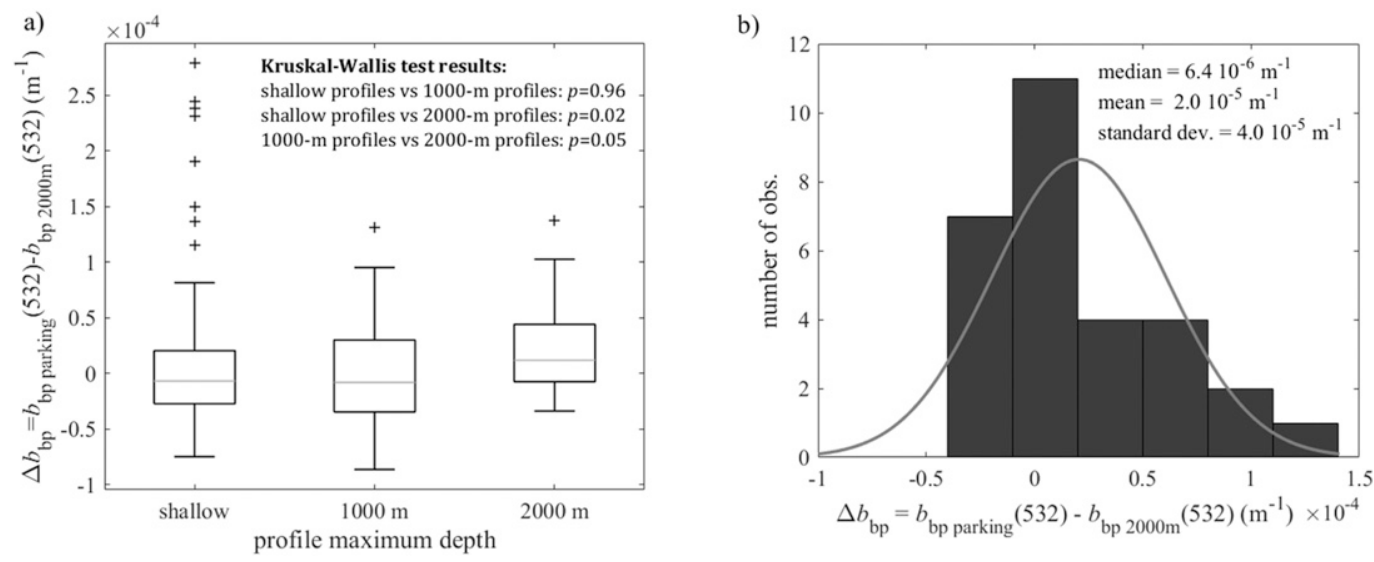

FIG. 7. Differences in the backscattering coefficient measured at the parking depth and at the beginning of the profile. (a) Differences for all profiles grouped based on the depth of the profile: the boxes indicate the range between $25 \%$ and $75 \%$ quartiles (Q25\% and Q75\%, respectively), and the plus signs indicate outliers. (b) Histogram of the differences recorded for deep profiles only.

value for $b_{\mathrm{bp}}(532)$ in the subtropical Indian Ocean or the linearity of sensor response with profiling depth. We do not recommend this method as a general postprocessing step, nor do we expect that it would necessarily work for other instruments, as we suspect this is a function of a specific defect of the individual instrument. However, it is likely that as the number of autonomous floats and sensors increases, this analytical approach could be used for quality assurance and correction of specific datasets. First, we assumed the initial values recorded by the a)

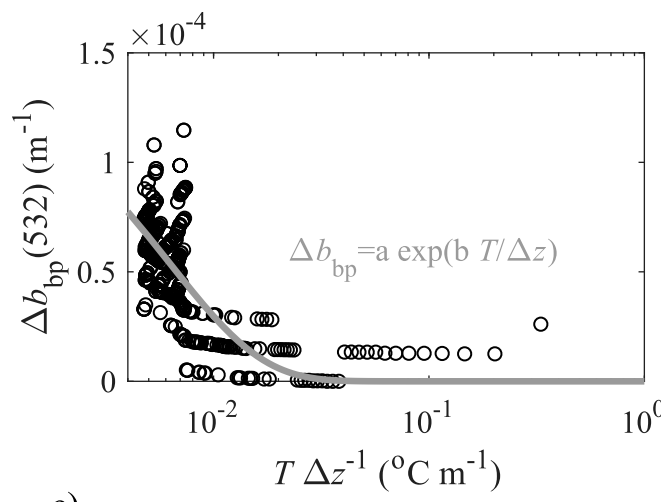

b)

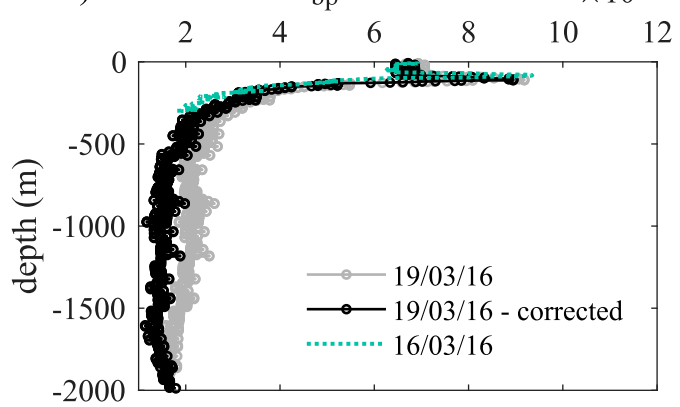

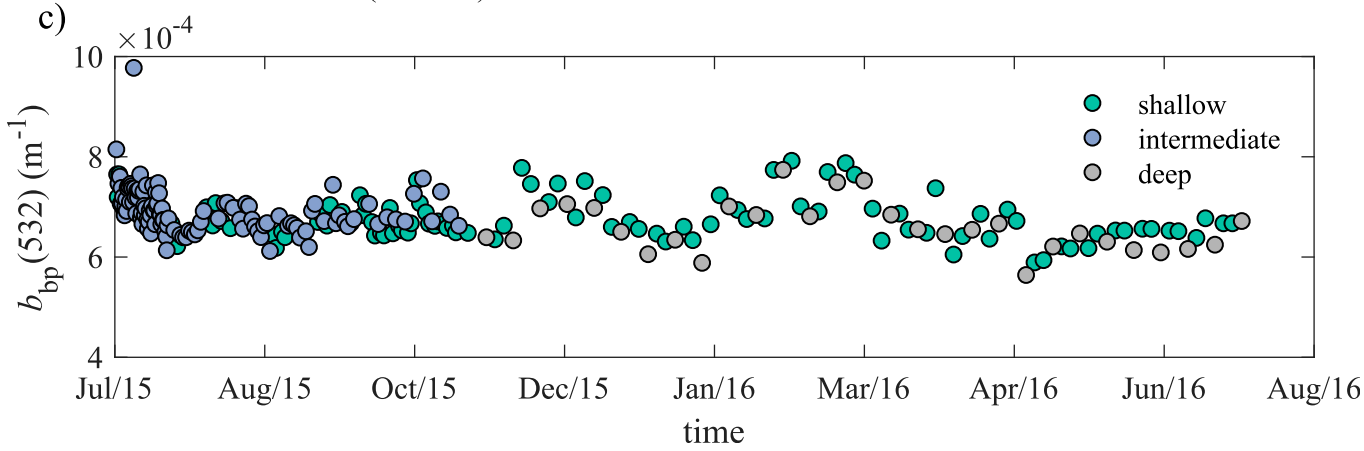

FIG. 8. Processing of the BGC-Argo float-derived backscattering coefficient profiles. (a) Derivation of the correction based on the first $1000 \mathrm{~m}$ of the profile. (b) Corrected and raw profiles of $b_{\mathrm{bp}}(532)$ measured on 19 Mar 2016 together with the shallow profile of $b_{\mathrm{bp}}(532)$ from 16 Mar 2016. (c) Time series of the surface values of $b_{\text {bp }}(532)$. 
sensor are "true." As mentioned before, this hypothesis is not yet verified, as it requires further investigation by the manufacturer, and we accept that an alternative hypothesis, that the sensor is initially reading low until it "warms up" could equally be valid. Second, we found strong exponential dependence $(r>0.8)$ between the increase in the backscattering coefficient and the ratio between temperature $T$ and distance traveled by the float $\Delta z$ from the start of the profile (Fig. 8a). This does not necessarily mean that the rate of increase is the same between consecutive profiles; for example, it could change as the float samples different water masses. Thus, we apply the correction of the affected profiles on a profile-by-profile basis. In the proposed correction method, the deepest parts of each profile are used to calculate the rate of increase in the backscattering signal caused by the anomalous sensor behavior. The thickness of the layer taken for calculations depends on the profiling depth and the shape of the backscattering profile itself. It is important to choose the part of the profile that is the least likely to be affected by real changes in the backscattering coefficient magnitude. In the example presented, we selected the initial (deepest) $1000 \mathrm{~m}$ of the profile. In the next step, this anomalous $b_{\mathrm{bp}}$ rate of increase with depth is subtracted from the entire profile (Fig. 8b). This approach enabled aligning the values of backscattering at within the water column (Fig. 8b) and at the surface (Fig. 8c) between profiles starting at various depth.

\section{Conclusions}

The effect described in this paper can significantly affect the values of the particulate backscattering coefficient derived from the MCOMS 532-nm volume scattering function measurement. We recommend including sampling at the parking depth and a calculation of the difference in the sensor-reported values of $b_{\mathrm{bp}}$ at the parking depth before and during profiling within a quality-assurance routine. By undertaking such checks, and by flagging the profiles possibly affected, the quality of $b_{\mathrm{bp}}$ data from autonomous platforms can be better controlled to assist in the generation of reliable, high-quality global datasets. Only one sensor exhibited the anomalous measurements, which suggests that while the data can be adjusted to align the profiles, we caution that this is not likely to be a general solution. However, a reasonably succinct ad hoc correction method was successful within this dataset in aligning multiple profiles and increasing the coherence of the profiles of the two backscattering sensors. Conclusive identification of the source of this sensor response requires laboratory experimentation to examine the behavior of the sensor under controlled conditions of temperature and pressure.

Acknowledgments. We thank the Argo team of the Integrated Marine Observing System (IMOS) for their technical support. All data are available online (ftp:// ftp.ifremer.fr/ifremer/argo/dac/csiro/; http://doi.org/ 10.17882/42182). We acknowledge the funding from the Australian government through the Australia-India Strategic Research Fund (AISRF) and CSIRO OCE Postdoctoral Fellowship support for B. W. We thank Giorgio Dall'Olmo and two anonymous reviewers for their useful comments and suggestions.

\section{REFERENCES}

Antoine, D., D. A. Siegel, T. Kostadinov, S. Maritorena, N. B. Nelson, B. Gentili, V. Vellucci, and N. Guillocheau, 2011: Variability in optical particle backscattering in contrasting bio-optical oceanic regimes. Limnol. Oceanogr., 56, 955-973, https://doi.org/10.4319/lo.2011.56.3.0955.

Boss, E., D. Bowers, L. Taylor, P. Brickley, R. Zaneveld, M. J. Perry, and P. G. Strutton, 2008: Observations of pigment and particle distributions in the western North Atlantic from an autonomous float and ocean color satellite. Limnol. Oceanogr., 53, 2112-2122, https://doi.org/10.4319/ lo.2008.53.5_part_2.2112.

Bricaud, A., C. Roesler, and J. R. V. Zaneveld, 1995: In situ methods for measuring the inherent optical properties of ocean waters. Limnol. Oceanogr., 40, 393-410, https://doi.org/ 10.4319/lo.1995.40.2.0393.

Briggs, N., M. J. Perry, I. Cetinić, C. Lee, E. D’Asaro, A. M. Gray, and E. Rehm, 2011: High-resolution observations of aggregate flux during a sub-polar North Atlantic spring bloom. Deep-Sea Res. I, 58, 1031-1039, https://doi.org/10.1016/j.dsr.2011.07.007.

Cetinić, I., M. J. Perry, N. T. Briggs, E. Kallin, E. A. D'Asaro, and C. M. Lee, 2012: Particulate organic carbon and inherent optical properties during 2008 North Atlantic Bloom Experiment. J. Geophys. Res., 117, C06028, https://doi.org/10.1029/ 2011JC007771.

Dall'Olmo, G., and K. A. Mork, 2014: Carbon export by small particles in the Norwegian Sea. Geophys. Res. Lett., 41, 29212927, https://doi.org/10.1002/2014GL059244.

- E. Boss, M. J. Behrenfeld, and T. K. Westberry, 2012: Particulate optical scattering coefficients along an Atlantic Meridional Transect. Opt. Express, 20, 21 532-21 551, https:// doi.org/10.1364/OE.20.021532.

Gardner, W. D., I. D. Walsh, and M. J. Richardson, 1993: Biophysical forcing of particle production and distribution during a spring bloom in the North Atlantic. Deep-Sea Res. II, 40, 171-195, https://doi.org/10.1016/0967-0645(93)90012-C.

Green, R. E., A. S. Bower, and A. Lugo-Fernández, 2014: First autonomous bio-optical profiling float in the Gulf of Mexico reveals dynamic biogeochemistry in deep waters. PLOS ONE, 9, e101658, https://doi.org/10.1371/journal.pone.0101658.

Johnson, K. S., and Coauthors, 2017: Biogeochemical sensor performance in the SOCCOM profiling float array. J. Geophys. Res. Oceans, 122, 6416-6436, https://doi.org/10.1002/ 2017JC012838. 
Maffione, R. A., and D. R. Dana, 1997: Instruments and methods for measuring the backward-scattering coefficient of ocean waters. Appl. Opt., 36, 6057-6067, https://doi.org/10.1364/AO.36.006057.

Mignot, A., H. Claustre, J. Uitz, A. Poteau, F. D’Ortenzio, and X. Xing, 2014: Understanding the seasonal dynamics of phytoplankton biomass and the deep chlorophyll maximum in oligotrophic environments: A Bio-Argo float investigation. Global Biogeochem. Cycles, 28, 856-876, https://doi.org/10.1002/ 2013 GB004781.

Poteau, A., E. Boss, and H. Claustre, 2017: Particulate concentration and seasonal dynamics in the mesopelagic ocean based on the backscattering coefficient measured with Biogeochemical-Argo floats. Geophys. Res. Lett., 44, 69336939, https://doi.org/10.1002/2017GL073949.

Riser, S. C., and K. S. Johnson, 2008: Net production of oxygen in the subtropical ocean. Nature, 451, 323-325, https://doi.org/ 10.1038/nature06441.

Stramski, D., E. Boss, D. Bogucki, and K. J. Voss, 2004: The role of seawater constituents in light backscattering in the ocean. Prog. Oceanogr., 61, 27-56, https://doi.org/10.1016/ j.pocean.2004.07.001.

Sullivan, J. M., M. S. Twardowski, J. R. V. Zaneveld, and C. C. Moore, 2013: Measuring optical backscattering in water. Light Scattering Reviews 7, Springer Praxis Books, Springer, 189224, https://doi.org/10.1007/978-3-642-21907-8_6.

Walsh, I. D., S. P. Chung, M. J. Richardson, and W. D. Gardner, 1995: The diel cycle in the integrated particle load in the equatorial Pacific: A comparison with primary production. Deep-Sea Res. II, 42, 465-477, https://doi.org/10.1016/0967-0645(95)00030-T.

Wojtasiewicz, B., N. J. Hardman-Mountford, F. Dufois, J. Greenwood, D. Slawinski, D. Antoine, and T. W. Trull, 2016: Relation between phytoplankton chlorophyll anad particulate organic carbon in the Indian Ocean. Proc. Ocean Optics XXIII, Victoria, BC, Canada, Oceanography Society.

Zhang, X., L. Hu, and M.-X. He, 2009: Scattering by pure seawater: Effect of salinity. Opt. Express, 17, 5698-5710, https://doi.org/ 10.1364/OE.17.005698. 